\title{
NUMERICAL SIMULATION ON A DEEP-DRAFT SEMI-SUBMERSIBLE
}

\author{
ALIATULNAJIHA AYUB AND MOHD ASAMUDIN A RAHMAN*
}

Faculty of Ocean Engineering Technology and Informatics, Universiti Malaysia Terengganu, 21030 Kuala Nerus, Terengganu, Malaysia

*Corresponding author: mohdasamudin@umt.edu.my

http://doi.org/10.46754/umtjur.2021.04.008

\begin{abstract}
A numerical study is conducted to determine the Vortex Induced Motion (VIM) effects on Deep-Draft Semi-Submersibles (DDSS). The VIM phenomena is a crucial problem that can cause severe impact on the fatigue life of mooring risers in DDSS. Therefore, a comprehensive numerical simulation is conducted using the Acusolve computational fluid dynamics (CFD) software. Five models of immersed columns with different aspect ratios (ie. 0.6, 0.8, 1.0, 1.2 and 1.4) are numerically investigated under two different incidence angles, which are $0^{\circ}$ and $45^{\circ}$. The transverse and in-line vibration amplitude, amplitude of lift force coefficient and vortex shedding are analyzed. The numerical measurements are obtained to see the response of horizontal plane motions, which are transverse, in line and yaw motions. This study with detailed numerical results from parametric data will contribute future studies and the comparisons are made to demonstrate the capability of the present CFD approach.
\end{abstract}

Keywords: CFD, VIM, DDSS

\section{Introduction}

As oil and gas exploration increases in deep waters, the Vortex Induced Motion (VIM) acting on an increasing number of floating platforms at sea will also become a main problem. The deepdraft semi-submersible (DDSS) is a marine vessel that is commonly used in the exploration of oil and gas in oceans, specifically as offshore drilling rigs, safety vessels and also a production facility.

More of these platforms have been fabricated and installed in deep waters, such as the Gulf of Mexico off the United States (Liang et al., 2017). They usually consist of four vertical cylindrical columns connected to the deck and lower pontoon type members. When the sea current flows through the columns, the resulting VIM will be generated from exciting forces caused by vortex shedding on a large floating structure, which will also generate strong cyclic dynamic effects on the floaters (Goncalves et al., 2010). It also provides the vortex shedding frequency when the harmonic oscillatory motion is almost in a constant amplitude and near-identical zero crossing periods, and this is known as the "lockin" phenomena.

VIM lock-in may occur under the right combination of mooring line stiffness, vessel mass and current speed. The effects of VIM has been investigated through various characteristics, and one of the studies is on different designs of the Spar platform. Finn et al. (2003) stated that when the model Spar platforms straked perfectly and have wise clean surface, they will have very small vortex-induced vibration in the presence of strong loop currents. Figure 1 shows a typical DDSS while Figure 2 shows the pattern of VIM acting on an eight-columned semi-submersible. 


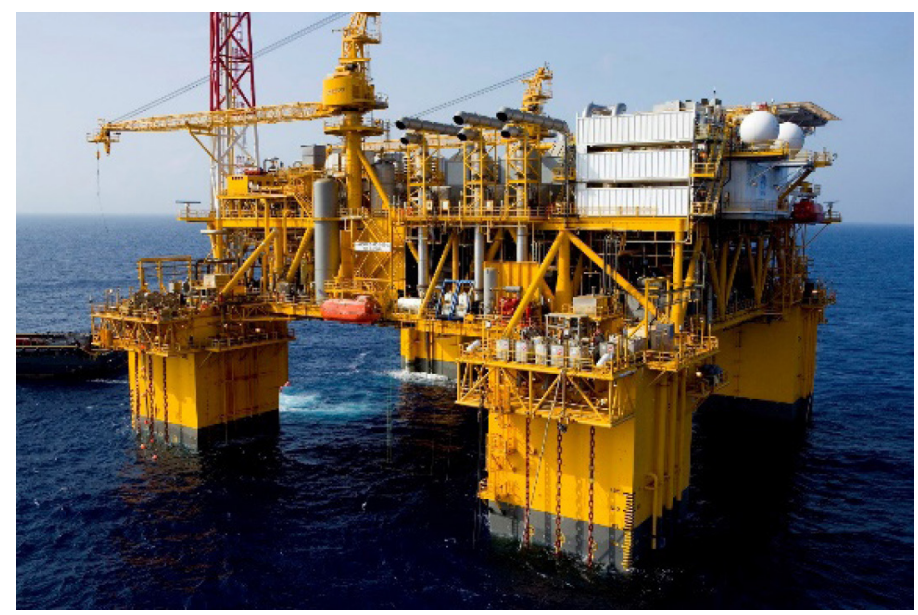

Figure 1: Typical deep-draft semi-submersible

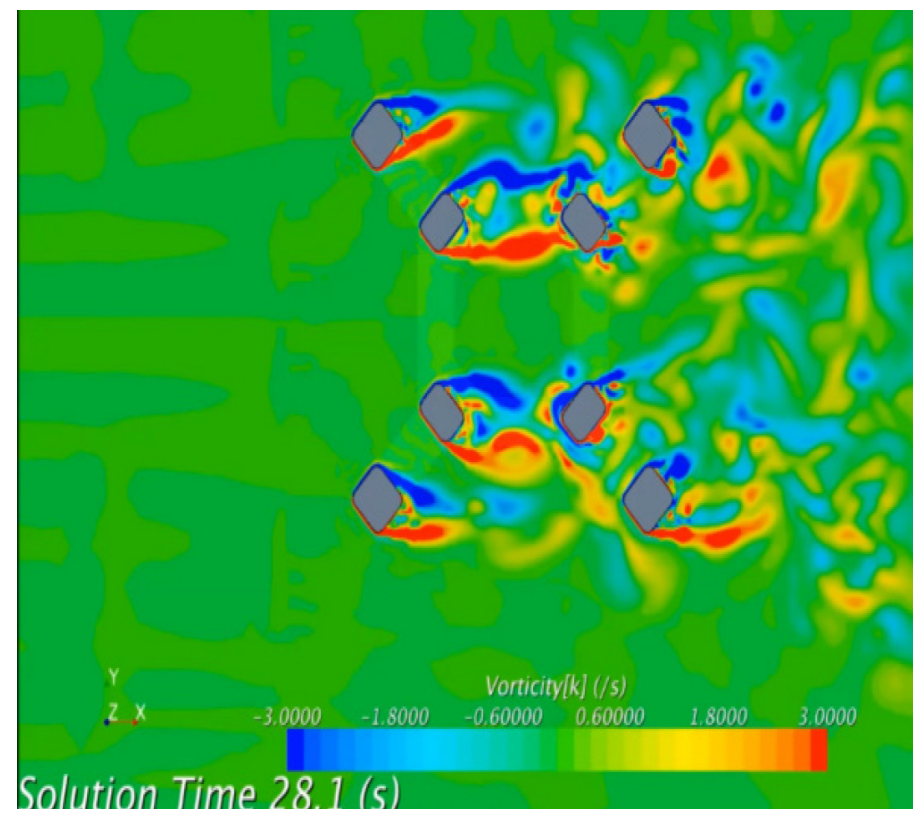

Figure 2: Vortex Induced Motion acting on an eight-columned semi-submersible

VIM is commonly referred as a selfexcitation phenomenon that may be observed on immersed bluff bodies (Liu et al., 2016). The phenomena is free to oscillate in a specific fluid flow condition, and the transversal section of the system can present the magnitude in amplitude values. VIM studies have been carried out mainly on spar platforms early in the decade.
This is because VIM causes great drift on the surface of platforms until it affects the mooring and riser systems, causing both extreme tension and fatigue on the vessel's structure. However, recently, research has been focusing on monocolumn platforms to minimise the problem (Antony et al., 2015; Kim et al., 2018). There is also a study that confirmed the effects of VIM on semi submersibles (Kang et al., 2015). 
One of the characteristics of floating platforms that makes it susceptible to VIM phenomena is their low aspect ratio (Norberg, 1993). The phenomena also have been noticed when the draft dimension of the columns increased, specifically the immersed aspect ratios. This is due to vortex shedding around the columns (Zhu \& Ou, 2017). Tests have been carried out on cylinders with low aspect ratios to compare with cylinders with high aspect ratios. The results showed variations in the motion amplitudes as the cylinders are investigated with two degrees of freedom (DOF) and the Strouhal Number ( $\mathrm{St}$ ) was observed based on the different aspect ratios. The study by Odijie et al. (2017) showed that decrease in draft conditions indicated the lower aspect ratio has contributed to the alleviation of VIM on the mono-column platform, and mono-Br floating unit.

\section{Materials and Methods}

\section{Parametric Study}

Parameters that were observed in five case studies stated in Table 1. These five case studies had different aspect ratios of their immersed column height above the pontoon, but the incidence angles were the same, which were set at $0^{\circ} \& 45^{\circ}$.

Table 1: Parametric study

\begin{tabular}{ccc}
\hline Case Study & Incidence Angle (degree, ${ }^{\circ}$ ) & Aspect Ratio (H/L) \\
\hline I & $0^{\circ} \& 45^{\circ}$ & 0.6 \\
II & $0^{\circ} \& 45^{\circ}$ & 0.8 \\
III & $0^{\circ} \& 45^{\circ}$ & 1.0 \\
IV & $0^{\circ} \& 45^{\circ}$ & 1.2 \\
V & $0^{\circ} \& 45^{\circ}$ & 1.4 \\
\hline
\end{tabular}

\section{Design Phase}

Rhino 6, a computer aided design (CAD) software, was used to design the DDSS. The model structure that has its own dimensions was combined together with the domain to run the stimulation.

\section{Deep-Draft Semi-Submersibles}

In Table 2 shown the specification and the dimension of the DDSS that has been designed by Rhino 6 . The dimension of the DDSS model with different aspect ratios were calculated as shown in Table 3. The characteristics dimension of the DDSS that had been used in previous simulation is shown in Figure 4. Next, Figure 5 shows the workspace of Rhino 6 that was used in designing the model.

Table 2: Dimension of the DDSS

\begin{tabular}{lc}
\hline Parameter & Value $(\mathbf{m})$ \\
\hline Distance between Centre Columns $(S)$ & 1.133 \\
Column Width $(L)$ & 0.305 \\
Immersed column height above the pontoon $(H)$ & 0.578 \\
Pontoon Height $(P)$ & 0.156 \\
Column Corner Radius $(\mathrm{R} c)$ & 0.047 \\
Pontoon Width $(\mathrm{L} p)$ & 0.156 \\
Pontoon Corner Radius $(\mathrm{R} p)$ & 0.031 \\
\hline
\end{tabular}


Table 3: Dimensions of the DDSS model tested with different draft conditions

\begin{tabular}{|c|c|c|c|c|c|}
\hline \multirow{2}{*}{ Parameter (m) } & \multicolumn{5}{|c|}{ Case Study } \\
\hline & I & II & III & IV & $\mathbf{V}$ \\
\hline Aspect ratio of the immersed column $(\mathrm{H} / \mathrm{L})$ & 0.6 & 0.8 & 1.0 & 1.2 & 1.4 \\
\hline Immersed column height above the pontoon $(H)$ & 0.183 & 0.244 & 0.305 & 0.366 & 0.427 \\
\hline Draft $(H+P)$ & 0.339 & 0.400 & 0.461 & 0.522 & 0.583 \\
\hline
\end{tabular}

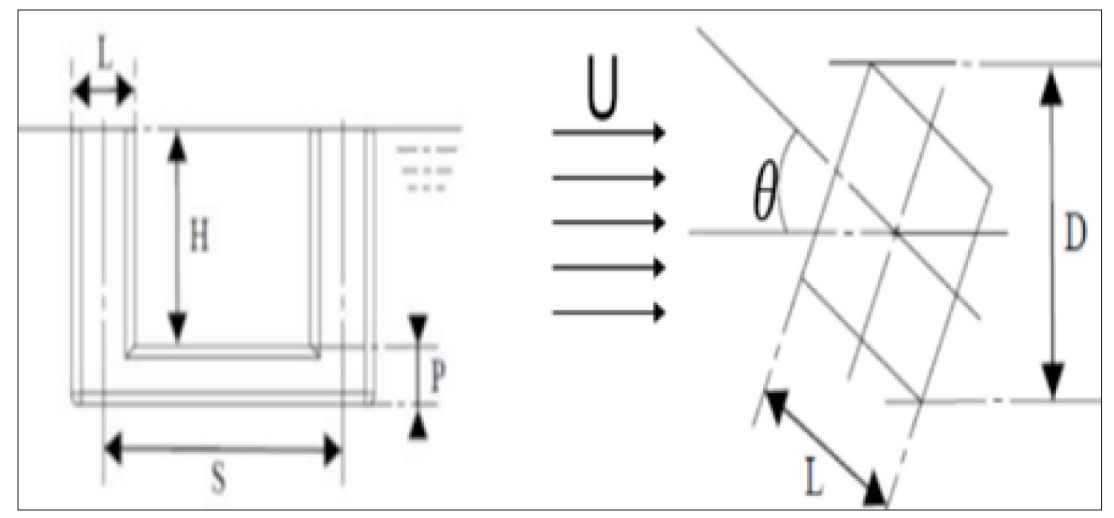

Figure 3: Characteristics dimensions of a DDS (Liang \& Tao, 2018)

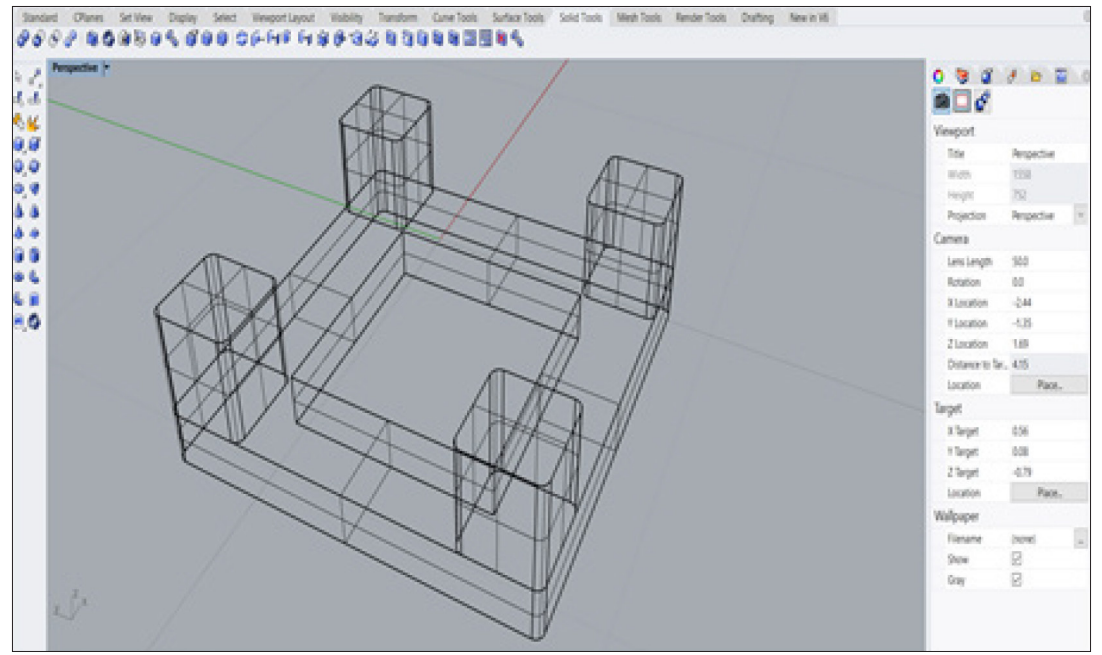

Figure 4: Design of the DDSS with H/L, 1.0 by using Rhino 6

\section{Domain}

Table 4 shows the dimensions of the domain used in the case studies, while in Figure 6 shows the geometry with specification in Rhino 6. To eliminate far field effects from the boundaries which affects the flow around the model, a domain of $25 \mathrm{~m} \times 9 \mathrm{~m} \times 3 \mathrm{~m}$ was selected. In addition to that, the three-dimensional effects 
from a span wise-cross flow direction could also be eliminated with these computational domain settings. This was shown by the computational domain settings used in Lee et al. (2014), which were $6 \mathrm{Bl} \times 4.5 \mathrm{Bl} \times 2.8 \mathrm{Bt}$ and $5 \mathrm{Bl} \times 4 \mathrm{Bl} \times 2.2 \mathrm{Bt}$. Settings that were considered large enough to do the elimination were $27 \mathrm{Bl} \times 18 \mathrm{Bl} \times 6.5 \mathrm{Bt}$ and $11 \mathrm{Bl} \times 6 \mathrm{Bl} \times 3 \mathrm{Bt}$, respectively.

Table 4: Dimensions of the domain

\begin{tabular}{cc}
\hline Parameter & Full Scale Dimension (m) \\
\hline Length & 25.0 \\
Width & 9.0 \\
Depth & 3.0 \\
\hline
\end{tabular}

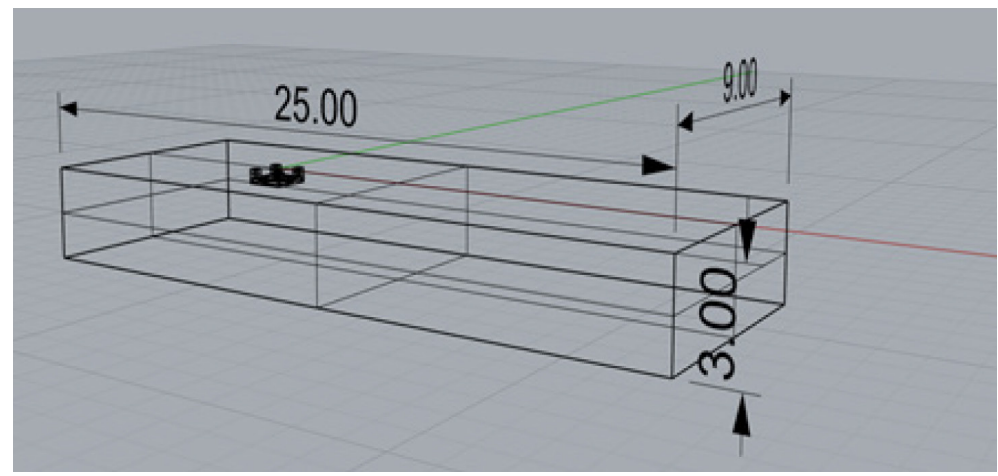

Figure 6: DDSS design with the dimensions using Rhino 6

\section{Boundary Condition}

In this phase, every geometry of the case studies was generated using the FLOW 3D software to extract their mass properties. The geometries were then imported into the AcuConsole. The Large Eddy Simulation (LES) was used because it was more accurate since large eddies contained most of the turbulent energy in sea currents. It is also responsible for most of the momentum transfer and turbulent mixing. This enables LES to capture the eddies in full directly as they modelled the RANS approach.
By using LES, computational costs could be reduced significantly as the large-scale motions (large eddies) of turbulent flow were computed directly and only small scale (sub-grid scale) motions were modelled (Zhiyin, 2015).

The time step was set at $200 \mathrm{~s}$ with initial time increment of $0.01 \mathrm{~s}$ where the time steps to complete was 2,000. The volumes and the surface were assigned for each part of the geometry including boundary conditions. The details of the geometry with boundary conditions are shown in the Figure 7 and kept the same for every simulation. 


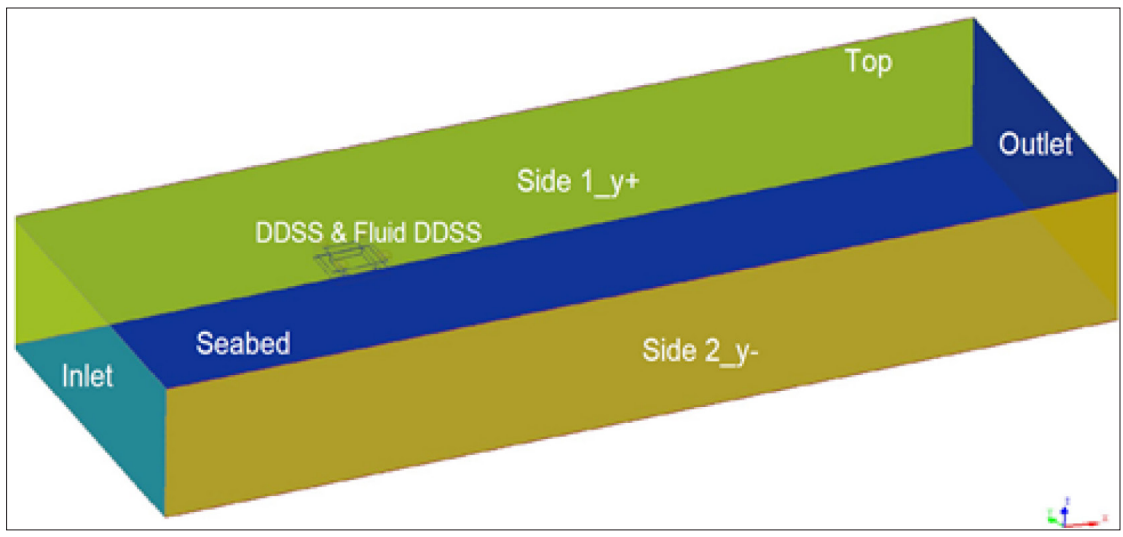

Figure 7: The volumes and surface for each part of the geometry after being assigned

\section{Results and Discussion}

Five case studies of immersed columns with aspect ratios $(\mathrm{H} / \mathrm{L})$ of $0.6,0.8,1.0,1.2$ and 1.4 were performed to determine the flow characteristics of vortex formation over the semi-submersible columns with different angles of incidence. Another purpose was to analyse the response of VIM, which consists of surge, sway and yaw motions that acted on the DDSS. The mean drag coefficient $\left(\mathrm{C}_{\mathrm{D}}^{-}\right)$and amplitude of lift coefficient $\left(\mathrm{C}_{\mathrm{L}}\right)$ were calculated for every simulation, including St. This section explained the results of the simulation.

\section{VIM Responses}

Mean surge amplitude

Figure 8 presents the results of the mean surge amplitude at incidence angles of $0^{\circ}$ and $45^{\circ}$. The in-line responses showed the highest surge amplitude at $\mathrm{H} / \mathrm{L} 1.4$ for both incidence angles, with peak values of $0.098 \mathrm{~m}$ and $0.012 \mathrm{~m}$, respectively. There was minimal fluctuation of surge amplitude in all $\mathrm{H} / \mathrm{L}$ of immersed columns tested at a $45^{\circ}$ incidence angle compared with $0^{\circ}$,

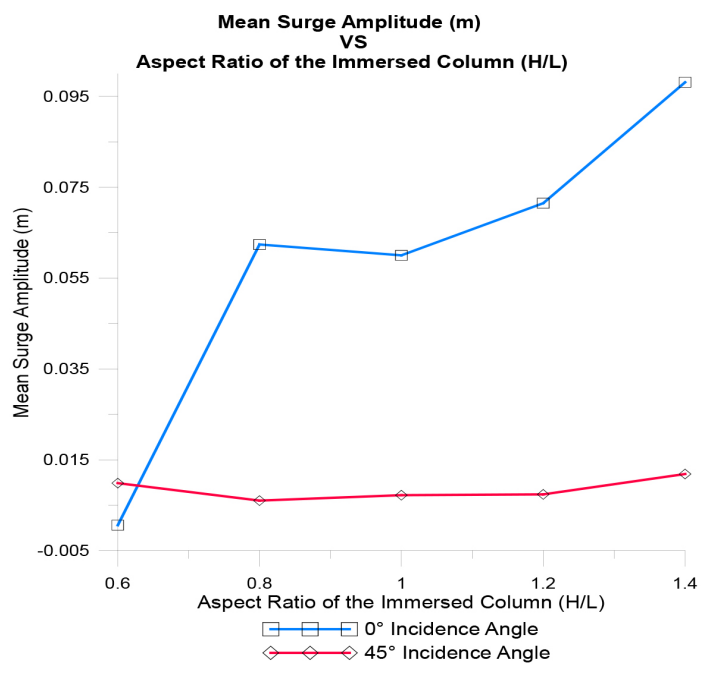

Figure 8: Surge motion amplitude on various aspect ratios of immersed DDSS columns 
Mean Sway Amplitude

In Figure 9, the highest mean sway amplitude at incidence angles of $0^{\circ}$ and $45^{\circ}$ for HL 1.4 were $0.030 \mathrm{~m}$ and $0.029 \mathrm{~m}$, respectively. Just like the surge amplitude, there was a sudden rise between HL 0.6 and 0.8 in the $0^{\circ}$ incidence angle curve. As the $\mathrm{H} / \mathrm{L}$ increased, the sway amplitude for $45^{\circ}$ incidence angle remained lower than $0^{\circ}$.

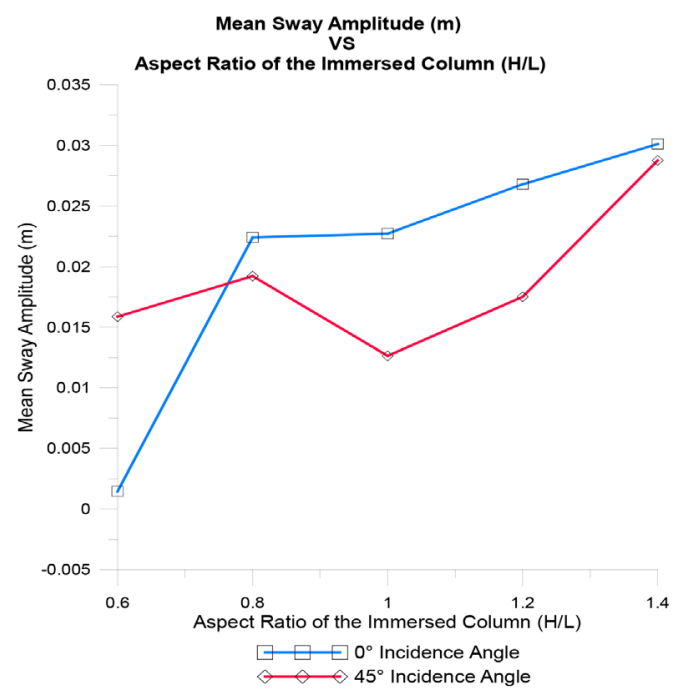

Figure 9: Sway motion amplitude on various aspect ratios of immersed DDSS columns

Mean Yaw Amplitude

Figure 10 shows The highest mean yaw amplitude for incidence angles of $0^{\circ}$ and $45^{\circ}$ were observed at H/L $1.2(0.036 \mathrm{rad})$ and $\mathrm{H} / \mathrm{L}$ $1.4(0.029 \mathrm{rad})$, respectively. The mean yaw amplitude was smallest at $0^{\circ}$ incidence angle.

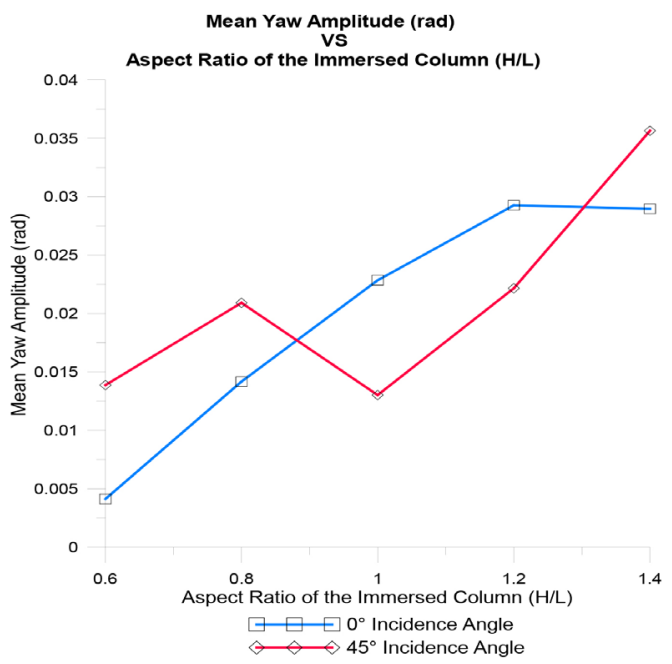

Figure 10: Yaw motion amplitude on various aspect ratios of immersed DDSS columns 


\section{Hydrodynamic Forces and Velocity Contour}

The hydrodynamics forces in the $\mathrm{x}$-direction and $y$-direction were normally represented in non-dimensional forms, namely the drag coefficient (CD) and lift coefficient (CL), which are represented in Equation (1) and Equation (2), respectively.

$$
\begin{aligned}
& C_{D}(t)=\frac{2 F_{x}(t)}{\rho A_{P} U^{2}} \\
& C_{L}(t)=\frac{2 F_{y}(t)}{\rho A_{P} U^{2}}
\end{aligned}
$$

where $\rho$ is the fluid density, Ap is the projected submerged area of the DDSS, Fx and Fy are the hydrodynamics force in the in-line and transverse directions, respectively, and $\mathrm{U}$ is the current speed at $0.25 \mathrm{~m} / \mathrm{s}$.

\section{Mean Drag Coefficient, $\left(\bar{C}_{D}\right)$}

Figure 11 shows the $\bar{C}_{D}$ where the peak for $0^{\circ}$ incidence angle was 2.02 , while for the $45^{\circ}$ incidence angle, it was 2.521 at $\mathrm{H} / \mathrm{L} 1.4$. The curves for both incidence angles showed an increasing trend, with $45^{\circ}$ having a sharper increase.

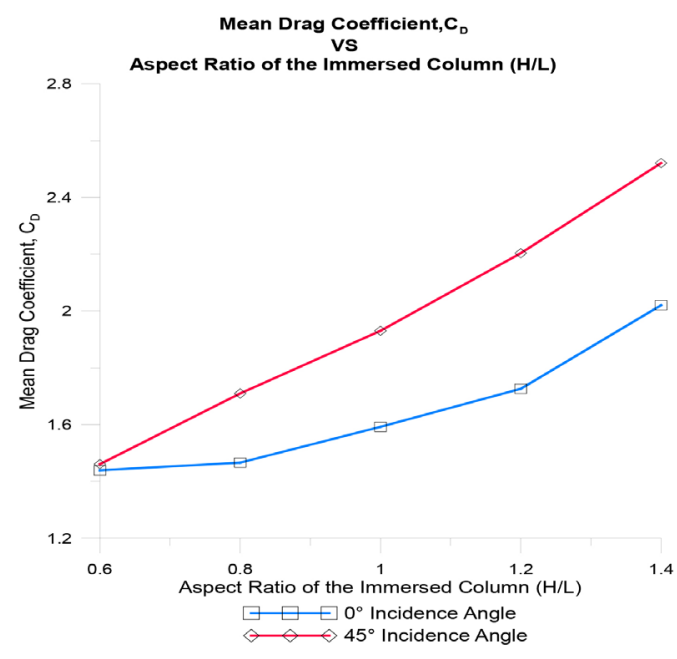

Figure 11: Mean drag coefficient

\section{Lift Coefficient Amplitude}

Figure 12 shows the ${ }_{L}$ curves for $0^{\circ}$ and $45^{\circ}$ incidence angles. The curve for $0^{\circ}$ incidence angle showed a drastic drop between H/L 1.0 and 1.2. The curve for $45^{\circ}$ incidence angle also showed a steady drop at $\mathrm{H} / \mathrm{L} 1.0$, but remained stable as $\mathrm{H} / \mathrm{L}$ increased. The peak values of amplitudes were 0.900 for $0^{\circ}$ incidence angle and 0.3225 for $45^{\circ}$ incidence angle. 


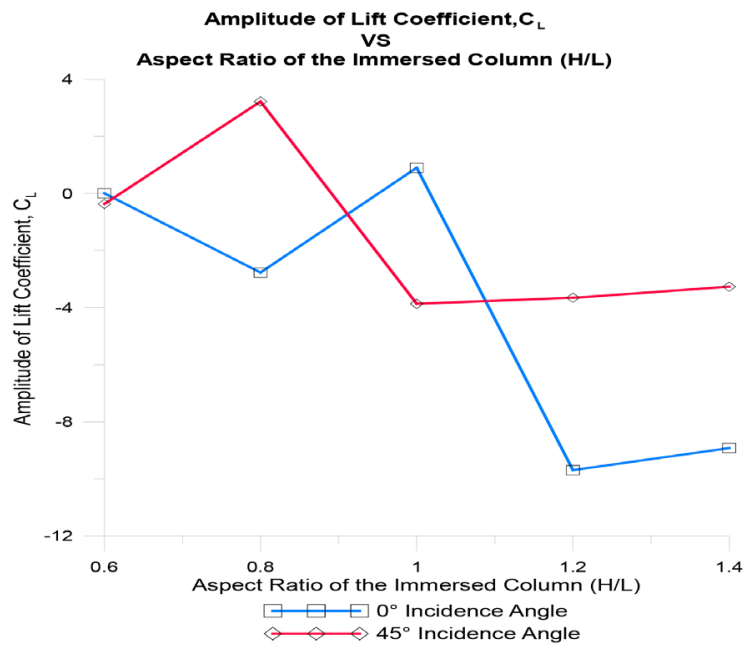

Figure 12: Lift coefficient amplitude

\section{Strouhal Number}

For $0^{\circ}$ and $45^{\circ}$ incidence angles, the St graph is shown for all $\mathrm{H} / \mathrm{L}$ values in Figure 13. Peak St for $0^{\circ}$ incidence angle was 0.265 , which was observed at $\mathrm{H} / \mathrm{L}$ 1.0. Meanwhile, for $45^{\circ}$ incidence angle, the highest value shown at $\mathrm{H} / \mathrm{L}$ 1.4, which was 0.063 , owing the largest St between the two incidence angle.

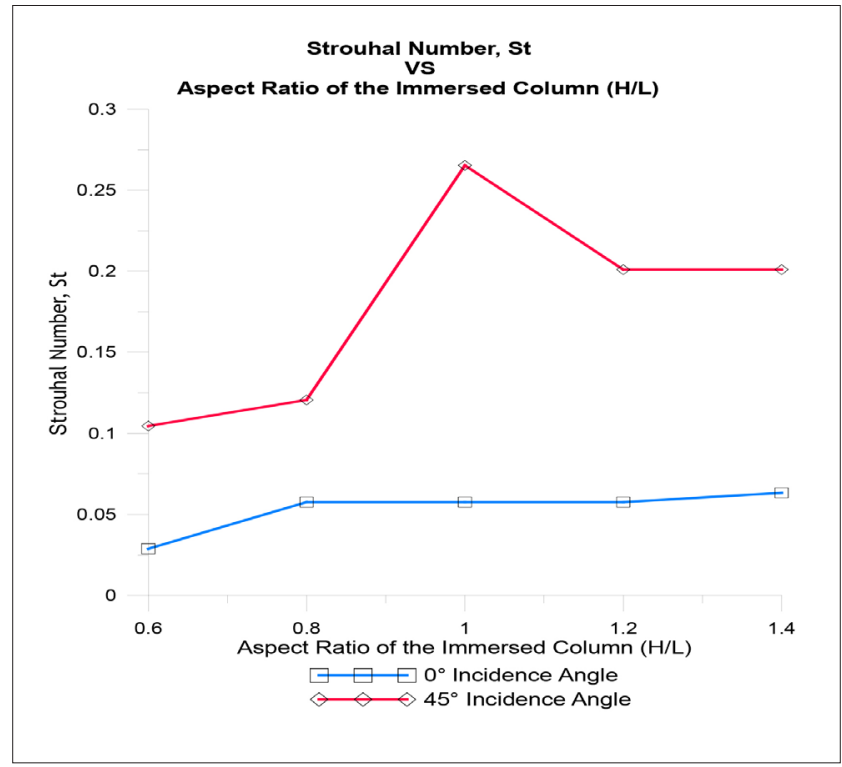

Figure 13: Strouhal number 


\section{Fast Fourier Transformation}

The Fast Fourier Transformation is a mathematical method that could be used to transform a function of time into frequency. It also describes the transformation of time to frequency domain. It is a practical formula to analyse time-dependent phenomenas. Table 5 shows the frequencies that have been extracted from every HL using Fast Fourier Transformation.

Table 5: Frequency of vortex shedding

\begin{tabular}{ccc}
\hline & \multicolumn{2}{c}{ Frequency } \\
\hline Aspect Ratio of The Immersed Column (H/L) & \multicolumn{2}{c}{ Incidence Angles $\mathbf{(}^{\circ}$ ) } \\
\cline { 2 - 3 } & $\mathbf{0}$ & $\mathbf{4 5}$ \\
\hline 0.6 & 0.005 & 0.013 \\
0.8 & 0.010 & 0.015 \\
1.2 & 0.010 & 0.033 \\
1.4 & 0.010 & 0.025 \\
\end{tabular}

\section{Velocity Contour}

The VIM response on DDSS with different aspect ratios were investigated at $0^{\circ}$ and $45^{\circ}$ incidence angle. As result, the vortex shedding occurs at the column corners for both heading $\left(0^{\circ}\right.$ and $\left.45^{\circ}\right)$ as expected. Figure 14 presents the flow visualization in terms of vorticity contours for every aspect ratio. These visualizations were taken at $\mathrm{T}=30 \mathrm{~s}$.

Based on Figure 14, at H/L 0.6, the vortex shedding produced was greater at $45^{\circ}$ incidence angle compared at $0^{\circ}$, where there was almost no vortex shedding occurring behind the platform. The yaw motion at $45^{\circ}$ incidence angle was also greater compared with $0^{\circ}$.

At $\mathrm{H} / \mathrm{L} 0.8$, vortex shedding at $0^{\circ}$ incidence angle produced a large wake along with a similarly large yaw response. At $45^{\circ}$ incidence angle, only a small yaw response could be seen, yet the vortex shedding produced behind the DDSS was large.

At $\mathrm{H} / \mathrm{L} 1.0$, the yaw response acting on the DDSS and magnitude of vorticity were huge for both incidence angles. Besides, at H/L1.2 for $0^{\circ}$ incidence angle, the yaw response acted slowly on the DDSS and the vortex shedding at the wake region was lesser compared with $45^{\circ}$ incidence angle. From the observation, there was almost no yaw response on the DDSS at $45^{\circ}$ incidence angle. Last but not least, at $\mathrm{H} / \mathrm{L}$ 1.4 , the vortex shedding for both incidence angles were greater at the wake region. At $0^{\circ}$ incidence angle, the yaw had a lot of noticeable violent swirls, which would cause the DDSS to drift, while there was almost no yaw motion was observed at $45^{\circ}$ incidence angle. 


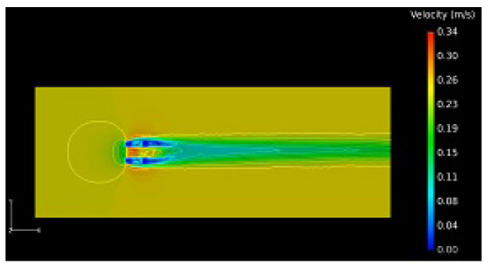

0.6
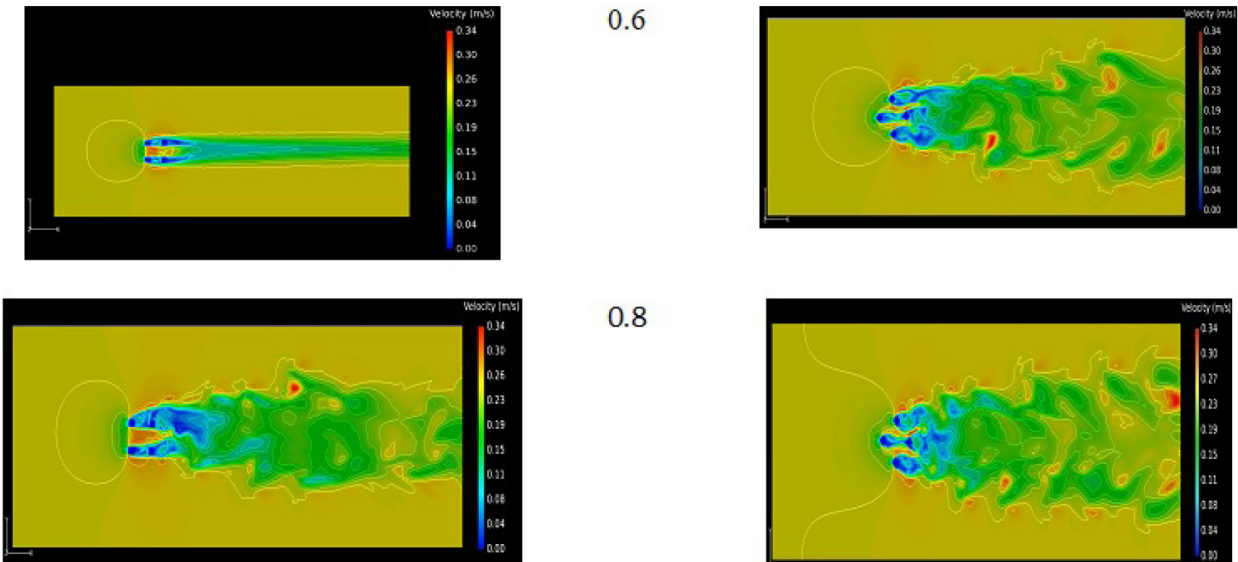

0.8
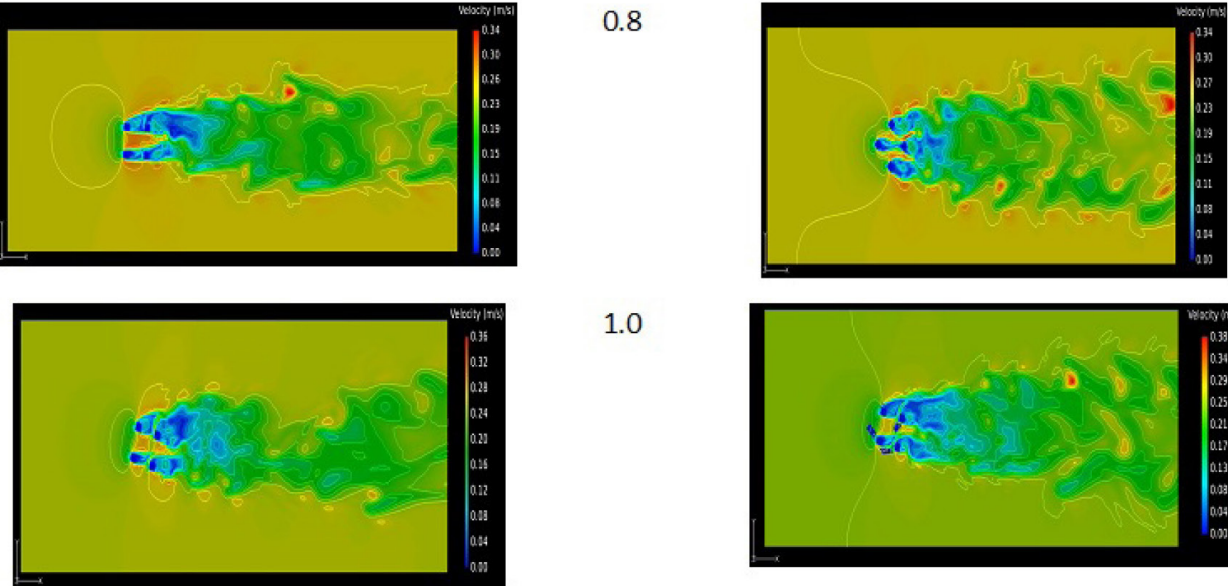

1.0
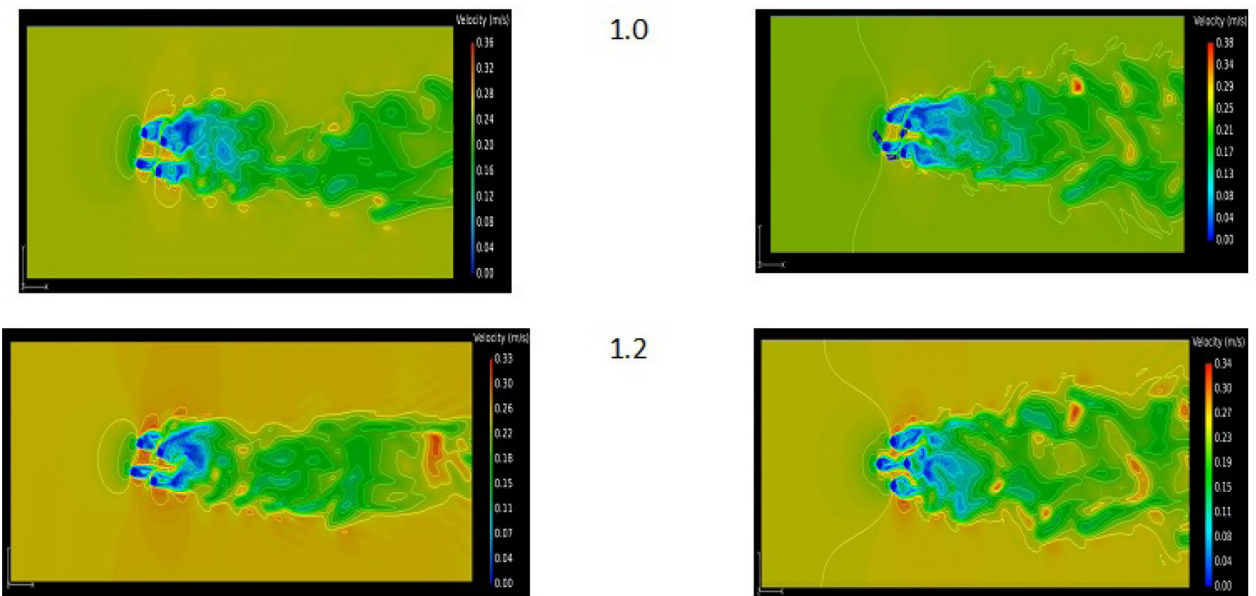

1.2
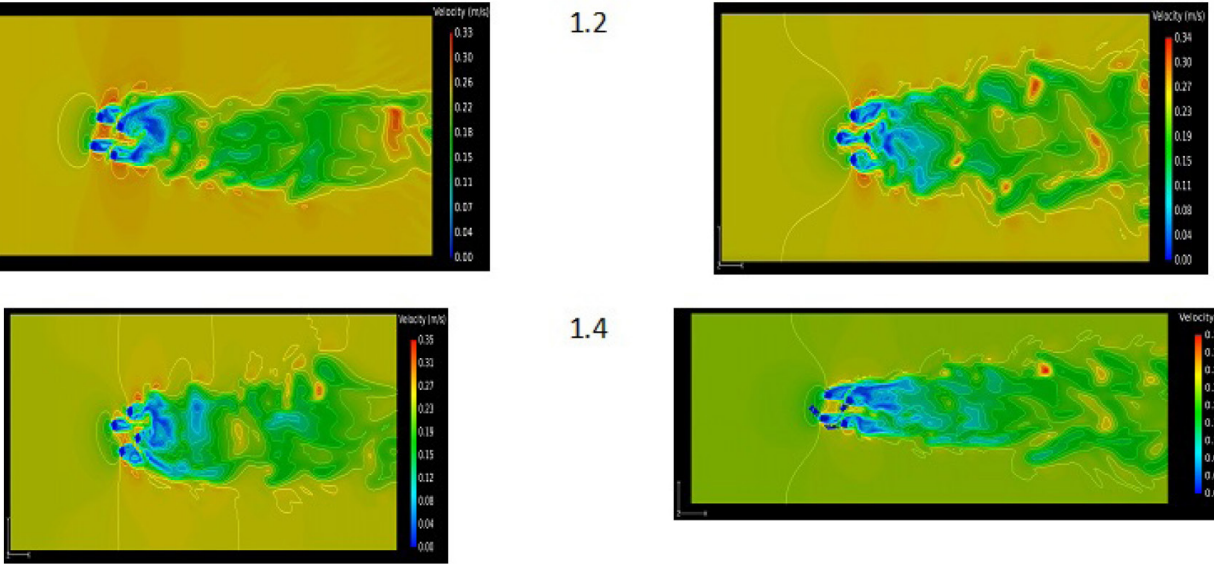

1.4

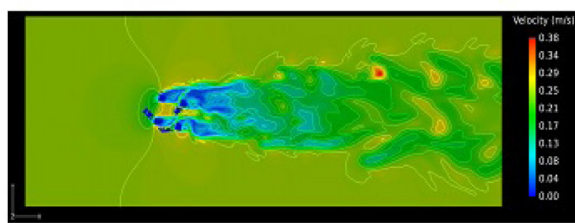

Figure 14: Flow visualization in terms of vorticity contours for every aspect ratio $(\mathrm{T}=30 \mathrm{~s})$

\section{Conclusion}

This numerical simulation was conducted to investigate the response of VIM in different draft conditions, and also to study the flow characteristics of vortex formation over semisubmersible columns with different incidence angles. Therefore, five aspect ratios of immersed columns were tested at incidence angles of $0^{\circ}$ and $45^{\circ}$. The aspect ratio of the DDSS columns play a very crucial part in the design of the vessel. This was because the VIM phenomena were clearly affected by the height of the DDSS columns. Surge motion was shown to be significantly higher at the incidence angle of $0^{\circ}$ compared to $45^{\circ}$. The most outstanding sway motion could be seen on both incidence angles, 
where the highest peak amplitude was at H/L 1.4. This varied from the lift force where at H/L 1.4, it was observed that the lowest amplitude was at $45^{\circ}$. For the yaw motion, the highest amplitude was observed at H/L 1.4 for an incidence angle of $45^{\circ}$. For $0^{\circ}$ incidence angle, where the highest peak was at H/L 1.0, the VIM response could be seen acting around the column vigorously.

\section{Acknowledgements}

The authors wish to thank the university for the research opportunity and the reviewers for the constructive comments.

\section{References}

Antony, A., Vinayan, V., Halkyard, J., Kim, S. J., Holmes, S., \& Spernjak, D. (2015). A CFD based analysis of the Vortex Induced Motion of deep-draft semisubmersibles. Proceedings of the International Offshore and Polar Engineering Conference, January, 1048-1055.

Finn, L. D., Maher, J. V., \& Gupta, H. (2003). The cell Spar and Vortex induced vibrations. Proceedings of the Annual Offshore Technology Conference, 2003-May, 16001605. https://doi.org/10.4043/15244-ms

Gonçalves, R. T., Fujarra, A. L. C., Rosetti, G. F., \& Nishimoto, K. (2010). Mitigation of vortex-induced motion (VIM) on a monocolumn platform: Forces and movements. Journal of Offshore Mechanics and Arctic Engineering, 132(4), 041102. https://doi.org/10.1115/1.4001440

Kang, Z., Ni, W., Zhang, L., \& Ma, G. (2017). An experimental study on vortex induced motion of a tethered cylinder in uniform flow. Ocean Engineering, 142(July), 259-267. https://doi.org/10.1016/j. oceaneng.2017.07.018

Kim, S. J., Spernjak, D., Mejia-Alvarez, R., Vinayan, V., Sterenborg, J., Antony, A., ... Halkyard, J. (2018). Numerical simulation of vortex-induced motion of a deep-draft paired-column semi-submersible offshore platform. Ocean Engineering, 149(May 2017), 291-304. https://doi.org/10.1016/j. oceaneng.2017.12.01

Lee, S. K., Chien, H. P., \& Gu, H. (2014). CFD study of deep draft semisubmersible VIM. Proceedings of the Annual Offshore Technology Conference, 1, 815-829.

Liang, Y., Tao, L., Xiao, L., \& Liu, M. (2017). Experimental and numerical study on vortex-induced motions of a deep-draft semi-submersible. Applied Ocean Research, 67, 169-187. https://doi.org/10.1016/j. apor.2017.07.00

Liu, M., Xiao, L., Lu, H., \& Shi, J. (2016). Experimental investigation into the influences of pontoon and column configuration on vortex-induced motions of deep-draft semi-submersibles. Ocean Engineering, 123, 262-277. https://doi. org/10.1016/j.oceaneng.2016.07.007

Norberg, C. (1993). Flow around rectangular cylinders: Pressure forces and wake frequencies. Journal of Wind Engineering and Industrial Aerodynamics, 49(1-3), 187-196. https://doi.org/10.1016/01676105(93)90014-F

Odijie, A. C., Quayle, S., \& Ye, J. (2017). Wave induced stress profile on a paired column semisubmersible hull formation for column reinforcement. Engineering Structures, 143, 77-90. https://doi.org/10.1016/j. engstruct.2017.04.013

Zhiyin, Y. (2015). Large-eddy simulation: Past, present and the future. Chinese Journal of Aeronautics, 28(1), 11-24. https://doi. org/10.1016/j.cja.2014.12.007

Zhu, H, \& Ou, J. (2017). ISOPE-17-041 Numerical simulation of the heave motion of a deep draft semi-submersible platform. International Society of Offshore and Polar Engineers, 32(5), 589-594. https://doi. org/10.3969/j.issn.1006-7043.2011.05.009 